\title{
Pathologico-Anatomic Categories of Choledochal End-Piece Stenosis Due to Chronic Pancreatitis and Clinical Significance
}

\author{
Yunfu Lv*, Xiaoguan Gong, Xiaoyu Han, Shunwu Chang, Ning Liu, Baochun Wang \\ Department of Surgery, People's Hospital of Hainan Province, Haikou, China \\ Email: 'yunfu_lv@126.com
}

Received May 26, 2013; revised June 26, 2013; accepted July 16, 2013

Copyright (C) 2013 Yunfu Lv et al. This is an open access article distributed under the Creative Commons Attribution License, which permits unrestricted use, distribution, and reproduction in any medium, provided the original work is properly cited.

\begin{abstract}
Background: Chronic pancreatitis caused by common bile duct segment stenosis is a common complication. It often results in near side bile duct expansion, bile drain disorder, appearing serious obstructive jaundice, biliary cirrhosis, lifethreatening. However, chronic pancreatitis causes not bravery manager narrow some light, some heavy, and the clinical manifestation is different too. We think there may be different kinds of pathological anatomy. As a result, we carried out the research of this subject. Objective: To investigate the anatomicopathological classification of terminal stenosis of the common bile duct (CBD) caused by chronic pancreatitis (CP) and the treatment. Method: A retrospective analysis was made for the management of sympatomatic stenosis of the terminal end of CBD 47 CP cases. Autopsy was performed in 25 bodies to verify our classification. Result: By analyzing operation and postoperative follow-ups to 47 patients with obvious choledochal dilatations (diameter $\geq 15 \mathrm{~mm}$ ) due to chronic pancreatitis, the authors have found that there exist three pathologico-anatomic categories of choledochal end-piece stenosis due to chronic pancreatitis. The stenosis of type I is the external-pressing annular stricture (59.6\%); type II is front wall of choledochus being compressed one (31.9\%); and type III is the pseudocystic oppression one (8.5\%). Conclusion: The treatment of CP patients complicated with terminal stenosis of CBD need individual consideration. Clinical Significance: Type I should be treated with biliary-enterostomy owing to more serious stricture (only No.3 the Bake's dilstors and smaller ones can be passed through its stenotic segment). Type II Could be managed with T-tube drainage because of its slighter stricture (Bake's dilators bigger than No.6 and No.12 French urinary catheter can get through the Choledochal terminal). If there aren't biliary and pancreatic complicated diseases, non-operative treatment can be carried out. Type III can undergo with the T-tube replacement between biliary tract and pseudocyst if pseudocystic decompression doesn't lead to obvious stenosis (type IIIo and IIIb). If type III combines type I, the internal drainage should be performed in both ectatic bile duct and cyst.
\end{abstract}

Keywords: Pancreatitis; Common Bile Duct Stenosis; Anatomicopathological Classification

\section{Introduction}

Chronic Pancreatitis (CP) is not rare in clinic. It's linked to alcohol damage, improper diet and bowel disease. Caused by the former is often referred to as alcohol CP, which is called gallstone caused by $\mathrm{CP}$. Alcohol $\mathrm{CP}$ in western countries is more, about $50 \%-76 \%$ of $\mathrm{CP}$ patients [1-3]. In some countries with higher living standards, high protein, high fat diet is the another important reason for the CP. In Asia, Africa and Latin America, on the other hand, some of the poorest countries and regions, such as India, cause of a diet low in protein and low fat

${ }^{*}$ Corresponding author. protein deficiency caused by $\mathrm{CP}$ accounts for about $28 \%$ to $100 \%$. At present our country although no bulk data report, but some cholelith disease and biliary tract disease is a common cause of CP [4]. $20 \%-30 \%$ CP can't find the exact etiology, known as chronic idiopathic pancreatitis.

$\mathrm{CP}$ divides into alcoholic, biligenic and idiopathic $\mathrm{CP}$ in sustained inflammation of the pancreas, pancreatic fibrosis substance morphology change and abdominal pain as the main characteristics, which all can lead to permanent damage to the pancreas secretion inside and outside, and a variety of complications. CP cause the end of the common bile duct stricture is a non-metabolic 
complications, and also one of common situations of biliary duct stenosis. So far, documented CP cause the end of the common bile duct stenosis which is more general, reported only one type, namely fixed circular narrow [5, 6]. The narrow, however, can not explain any CP concurrency in bile duct expansion, but surgery with Bake's dilator and French catheter probe, pancreatic biliary no significant stenosis of practical problems. With this problem which begins from 1991, the author have observed and studied 47 cases of CP complicated with bile duct expansion (diameter $\geq 15 \mathrm{~mm}$ ) and 25 cases of adult antopsy.

\section{Materials and Methods}

\subsection{Clinical Material}

The group of patients consisted of 26 males and 21 females, with 49.3 years old of mean age (range, 28 - 72 years old). All patients had elevated alkaline phosphatase (AKP) level (range, $136-1563 \mathrm{u} / \mathrm{L}$ ); 25 of these patients had elevated r-transpeptidase (r-GT) level; fifteen had jaundice (median value of serum total bilirubin, 90.6 umol/L; range, 25.3 - $165 \mathrm{umol} / \mathrm{L}$ ); and fifteen patients' fasting blood-glucose values were more than $6.1 \mathrm{mmol} / \mathrm{L}$. six patients had undergone cholangitis; forty-three suffered from epigastralgia; forty $(85.1 \%)$ complicated cholecystolithiasis and 7 cases with a history of being drunk. $61.7 \%$ of these patients experienced more than once the attack of acute pancreatitis. Diagnostic examinations, including B-ultrasonography examination to 47 patients, and simultaneously, computed tomography (CT) examination to 21 patients, endoscopic retrography cholangio pancreatography (ERCP) examination to 18 patients, and percutaneous transhepatic cholangiography (PTC) examination to 14 patients, all prompted that these patients had obvious intrahepatic and extrahepatic duct dilatations, and displayed that thirty-seven patients contracted chronic pancreatitis and five among them complicated pancreatic pseudocyst.

\subsection{Surgical Situation}

All patients had undergone operations owing to gallstones in 40 of them, pancreatic pseudocyst in 5, and explorations for obvious choledochal dilatation complicated with jaundice in 2 patients. In operation, we observed that thirty patients had notable swelling, cirrhosis pancreas; fourteen patients had a segmental swelling, fibrous head and body of pancreas; and three patients had a focal swelling, cirrhosis head of pancreas, including one carcerization. In 31 patients whose pancreatic tissues were excised in operation to make pathological examinations, except one carcinoma of head of pancreas, the others had been verified suffering from CP. All patients had undergone cholecystectomy and choledochotomy with exploration. In the course of operation, incising choledochus, except that three patients were found in their bile ducts three were 1 to 3 stone of size of willet, all the others weren't found stones, cysts, ascarides or tumors in intrahepatic and extrahepatic ducts, confirmed by the choledochoscope examination.

Only No. 1 to No. 3 Bake's dilators and the French urinary catheters below No. 10 could be passed through the choledochal end-piece in 28 patients whose choledochus traversed their pancreases, and the dilator's bougies could not be touched at the back of their pancreases. The type of choledochal stenosis has been known as type I, the external-pressing annular stricture (Figure 1). Operative procedure of this type is as following: two patients of them, one owing to carcerization and another owing to stones in the head of pancreas, had undergone pancreatoduodenostomy. The other 26 pantients were treated with internal drainage, including 11 cholecystojejunostomies, 12 Roux-en-Y cholecystojejunostomies and 3 Braun's anastomsis added to cholecystojejunostomies. Three patients with pancreatic duct dilatation (diamter, 8 - 12), two of them with stones in pancreatic duct and the other with pancreatic pseudocyst in the body and tail, had undergone internal drainage.

By comparison, No. 4 to No. 11 Bake's dialators and the French urinary catheters more than No. 12 could be passed through the choledochal end-piece in 15 patients, whose choledochus walked along the back of pancreas and posterior wall wasn't covered with pancreatic tissues. Therefore, the dilator's bougies could be touched at the back of their pancreases. We named it stenosis of type II, the compressed-front-wall of choledochal stenosis (Figure 2). Operative procedure of this type is as following: two had undergone Roux-en-Y cholecystojejunostomy; the other 13 were treated with T-tube drainage.

In remained 4 patients, their choledochal stenosis cause by pancreatic pseudocyst, which compressed their pancreas of head. We named it stenosis of type III, the pseudocyst-constrictal stenosis (Figure 3). Operative procedure of this type is as following: after their pseudocyst had been incised and decompressed, all 4 patients

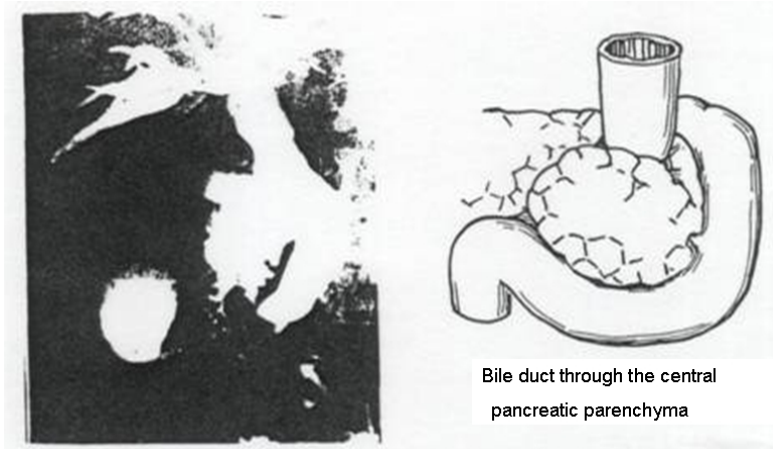

Figure 1. I Solid circular stricture. 
had undergone exploration; one patient without choledochal end-piece stenosis had experienced T-tube replacement added to cystojejunostomy. Two patients complicated with stenosis of type I had undergone double Renx-en-Y anastomosis (Figure 4), that is, cystojejunostomy and anastomosis between dilatative biliary duct and jejunum. The remained one complicated with stenosis of type II were treated with T-tube replacement in choledochus and Roux-en-Y pseudocystojejunostomy.

\subsection{Choledchal Dilatation Situation}

All patients' proximal choledochus had been found obvious dilatation. The diameter of proximal choledochus can be seen in Table 1.
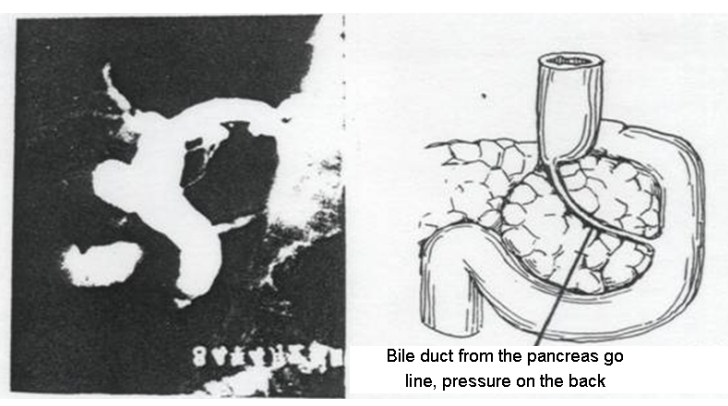

Figure 2. II Compression sex is relatively narrow.
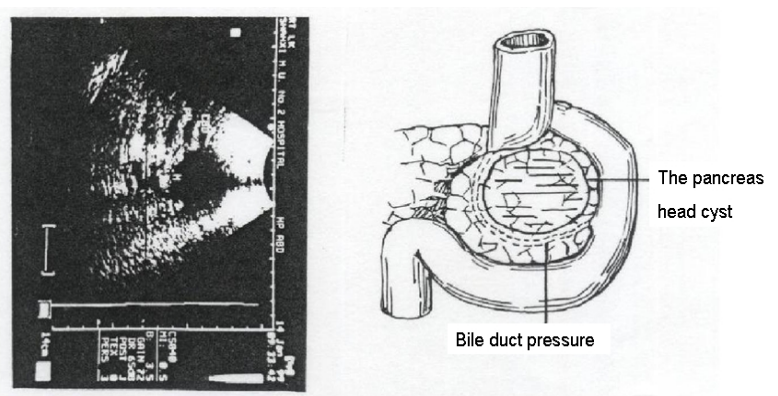

Figure 3. III cyst compression stenosis.

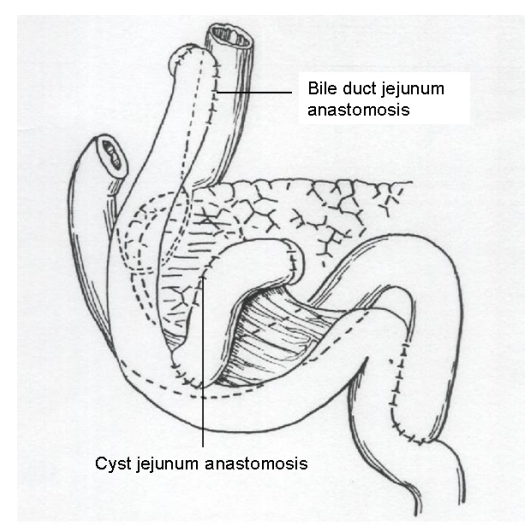

Figure 4. Expansion of the bile duct and cyst with jejunum anastomosis respectively, namely double Roux en-Y anastomosis.
Table 1. Proximal Choledochus diameter.

\begin{tabular}{cccc}
\hline \multirow{2}{*}{ type } & \multicolumn{2}{c}{ Diameter $(\mathrm{mm})$} & total \\
\cline { 2 - 3 } & $15-20$ & $21-25$ & \\
\hline I & 8 & 20 & 28 \\
II & 11 & 4 & 15 \\
III & 2 & 2 & 4 \\
total & 21 & 26 & 47 \\
\hline
\end{tabular}

\section{Statistical Analysis}

CBD and Akp values of stenosis type I are contrasted with type II by t-test or t'-test. After dealt with statistic package-SAS6.12, results are following:

Table 2. Compare parameters of CBD of type I \& II.

\begin{tabular}{ccccc}
\hline Type & case & $\bar{X} \pm \mathrm{S}(\mathrm{mm})$ & $\mathrm{t}$ & $\mathrm{p}$ \\
\hline $\mathrm{I}$ & 28 & $21.25 \pm 2.977$ & & \\
II & 15 & $18.067 \pm 2.987$ & 3.3381 & 0.0018 \\
\hline
\end{tabular}

Notes: These showed that CBD values of Type I are significantly different with that of type II, CBD of type surpassed type II.

Table 3. Compare parameter of AKP values of type I \& II.

\begin{tabular}{ccccc}
\hline Type & case & $\bar{X} \pm \mathrm{S}(\mu / \mathrm{L})$ & $\mathrm{t}$ & $\mathrm{p}$ \\
\hline I & 28 & $644.39 \pm 378.9651$ & & \\
II & 15 & $374.3667 \pm 198.187$ & 3.2902 & 0.0021 \\
\hline
\end{tabular}

Notes: these showed that AKP values of type I are significant different with type II.

\section{Results}

None in the group died from operation. 41 patients' abdominaliga symptom had disappeared. Re-examinations had been made after postoperatively 1 month, and results were as follows: AKP values in all patients had reduced to a mean value of $121 \mu / \mathrm{L}$. Serum total bilirubin values in 15 patients, which had elevated preoperatively, had given down to a mean value of $17.3 \mathrm{umol} / \mathrm{L}$. Postoperative follow-up surveys had been made, at the median follow-up of 42 months ranging from five months to eight years. With the exception of 11 patients who lose investigations, the other 36 (23 belonging to type I, 10 belonging to type II, 3 belonging to III) were obtained, and there is $76.6 \%$ of follow up rate. Follow-up results are as follows. Among those patients belonging to type I, two patients suffered from chornic reflux cholangitis after choledochoduodenostomy, whose symstoms had appeared at interval and could be controlled by medicine. One patient, who had treated with Roux-en-Y choledochojejunostomy, had undergone secondary operation (internal drainage) owing to stones and anastomotic stenosis. 
One patient with pancreatic cancerization who had undergonones after e pancreatico-duodenectomy had been dead after 31 months. Among those patients who belonged to type II, one had undergone Roux-en-Y choledochojejunostomy three years after the internal drainage, whose chronic pancreatitis had acute attacks many times and complicated jaccndice. Other patients had not untoward effect. Among those patients belonging to type III, one patient had a secondary internal drainage because of the stenosis of anastomotic stoma after operation, and the other 2 was normal.

\section{Autopsy}

To prove walks of common bile duct in pancreas, we had dissected 25 adult corpses, and found that common bile duct passed through pancreas in 17 of 25 corpses (accounting for $68 \%$ ). Common bile duct walked along the back of pancreas which posterior wall was not covered with pancreatic tissues in 8 corpses (accounting for 32\%). These approached with $61.3 \%$ and $38.7 \%$ reported by Fei and Ran [7], and showed that two ways of choledochal walk in pancreatic segment, which had been found in operation, was objective reality.

\section{Discussion}

CP has three kinds, that is, total, segmental, and local. Whichever has possibility to cause choledochal endpiece stenosis and lead to obvious dilatation in proximal bile duct, as long as leision implicates pancreatic head. Its incidence rate is $3 \%-46 \%$ in patients with CP [5,814]. The majority of the reports are about $25 \%$.

\subsection{Etiopathogenesis}

There are many reasons for choledochal end-piece stenosis due to chronic pancreatitis [15]. The followings are main reasons:

1) The edematous and tumescent pancreatic head crushs biliary duct to luminal stenosis. Especially in stage of acute attack, the stenosis can be exacerbated. After hydrops and hyperemia have extinguished, stenosis of bile duct can gradually be abaled, even disappeared. The process is reversible, and patients might have temporary jaundice, and it generally needn't surgery and could obtain natural cure.

2) The hyperplastic and fibroid pancreas which constrictes and pulls bile duct could lead to choledochal end-piece stenosis [16]. The process that can often be seen in the advanced stage of chronic pancreatitis is ireversible, and stenosis of bile duct is permanent. Fibrosis due to chronic pancreatitis could lead to not only choledochal end-piece stenosis, but also original part of pancreatic duct [17-19] and duodenum stenosis [13]. Huizinga et al. [20] had been proved by biopsy of liver that the kind of patients could still compilate hepatic portal fibrosis to different degrees.

3) Cyst compresses bile duct. Pancreatic pseudocyst in the body or tail could not generally lead to obstruction of biliary tract, but in the head could compress bile ducts to stenosis or make former stenosis segment exacerbate and prolong. Four of five pancneatic pseudocysts lie in the head, and three among them make former stenosis segment prolong.

\subsection{Diagnoses}

1) Diagnosis of chronic pancreatitis: Over 60 percent of patients with $\mathrm{CP}$ had a history of acute pancreatitis attack. A few patients, whose symptoms were latent, had always developed to chronic stage when found. $31 \%$ of patients complicated diabetes. Patients with different kinds of CP could still be questioned to different causes of illness. For example, patients with alcoholic CP had lasting excessive drunk at the same time. Patients with biliogenic $\mathrm{CP}$ often complicated illness of billary tract system, and so on. Epigastralgia is not sole characteristic of CP. Because patients with biliary tract illness could also have abdominal pain, moreover, $85 \%$ of patients had not it. Russoll et al. had instanced that nineteen patients had painless jaundice, and regarded as other kind of CP. Part of patients could appear the following symptoms: nausea, vomiting, fatty diarrhea, fatigue, indigestion, loss of body weight, and might still complicate pancreatic pseudocyst $(10.6 \%)$ or pancreatic duct stones $(6.4 \%)$. Kamal [15] insisted that diagnosis of CP could be tenable if there are the following condition over one: pancreas calification, dilatation in pancreatic duct as well as vanish of scondary and tertiary pancreatic duct, pathologic diagnosis by biomicroscopy. Prinz et al. [11] described detailedly the picuture of pancreatic duct system that were stenosis of initial segment of major pancreatic duct, dilatation in distal pancreatic duct, or multiple, segmental stenoses, and little branch blunt, distort, even disappear, which was in accordance with extensive lesion due to CP. Most of patients in the group could be seen calcified plaque and dilatation of pancreatic duct, and $66 \%$ of these patients had been extracted pancreatic tissues to do pathological examination, proved with $\mathrm{CP}$ except a pancreatic cancer.

2) Diagnosis of choledochal stenosis: To a patient with chdedochal dilatation, for the first time, B-ultrasonography, CT, ERCP, PTC and upper disgestive tract radiography with barium as well should be adopted to know whether there are stones, ascaris, tumor, congenital cholangiectasis, Constrictive papillitis, diverticultis of the bypass duouenal or pericholedochal tumor. If the above mentioned illnesses that can led to choledochal terminal obstruction could be ruled out and diagnosis of CP could be established, the obstruction should be considered due 
to $\mathrm{CP}$.

Clinical manifestations and pathological changes caused by obstruction of biliary tract are as follows: jaundice (31.9\%), cholangitis $(12.8 \%)$, biliary duct stone $(6.4 \%)$, biliary duct fibrosis (7.3\%) [13] as well as biliary cirrhosis of liver $(7.3 \%)$. As far as laboratory examination is concerned, AKP examination has comparatively higher diagnostic value and median AKP value of all patients in the group is 3.53 times as much as highest limit of normal value. Pereira-Lima et al. [11] maintained that, in the course of development of $\mathrm{CP}$, choledochal end-piece stenosis should be doubted if AKP value sustains on high level, no matter whether serum bilirubin raises or not. If AKP value is over three times as much as normal value, lasting over 1 month, it could be operation indication on choledochal stenosis. ERCP and PTC examinations are effective methods to make a definitive diagnosis [21]. Especially in development of pancreatic duct, ERCP have more distinctive value. The two methods can show whether there are stenosis, dilatation, stones as well as tumor, and can know state of intrahepatic and pancreatie duct. Stenotic segment of choledochus due to CP is usually longer than $34 \mathrm{~mm}(39 \pm 5 \mathrm{~mm})$, and most of them is smooth, symmetrical and grandually attenuated, a few unsmooth $[7,8]$. Diameter of dilatating proximal choledochus generally is within $25 \mathrm{~mm}$. B-ultrasonography and $\mathrm{CT}$ are the first choice of unautmatic examination, which could be used to measure diameters of bile and pancreatic duct, and to observe liuminalstate and figure of liver, bile and pancreatic system. Nomal choledochal diameter is 5 to $8 \mathrm{~mm}$, over $10 \mathrm{~mm}$, bile duct ditatation should be highly doubted by radiology. Nagazima et al. [22] thought if proximal choledochus dilated $\geq 15 \mathrm{~mm}$, or in operation, No. 3 Bake's dilator and No. 10 French urinary catheter could not be used to insert into duodenum from choledochal incision diagnosis of choledochal end-piece stenosis could be identified. Karmal et al. reported 19 cases with chdedochal end-piece stenosis whose median proximal choledochal diameter was $16 \mathrm{~mm}$. Newton et al. [23] thought that choledochal exploration by Bake's dilator and/or rubber urinary is common menthod to judge whether stenosis or not, which is simple and effective.

\subsection{Types}

According to exploration and autopsy findings, choledochal end-piece stenosis can be classified into three types.

Type I is the external-pressing annular stricture (59.6\%). The choledochus of the type runs cross pancreas. The swelling and fibrous panereas due to inflamation crushs circularly bile duct to stenosis, which only could be, passed through the Bake's dilators smaller than No. 3 and the French urinary catheters smaller than No. 10.
And in our choledochal explorations, Bake's dilator bougies could not be touched at the back of the pancreas.

Type II is the front wall of choledochus being compressed stenosis $(31.9 \%)$. The common bile duct of the type walks closely along the back of pancreas. The swelling and fibrous pancreas due to inflamation compresses the anterior wall of choledochus [24], so that it gets relatively stenosis. However, without pancreatic tissues covering the posterior wall of common bile duct, duct wall has better elasticity. So the bake's dilators more than No. 4 , even No. 8 to No. 11 Bake's dilators and more than No. 12 French urinary catheters in few patients, could be passed gradually through it. It seems not to be a stenosis. But radiological examinations showed obvious dilatation in proximal bile duct. And the authors found in operation that bile ducts had been pressed flat by pancreas. All these showed that there was stenosis in the choledochal end-piece. The stenosis of the type is mainly judged by operation. The procedure to explorate choledochal end-piece is as follows: first, cutting out part of the duodenal sie peritoneum, secondiy, turning over the duodenum to observe condition of bile duct, whether walking along the back of pancreas and whether being compressed, then, inserting the fore finger and middle finger of surgeon's left hand to the back of the patient's pancreas, holding Bake's metal dilator by right hand which had been crooked to a certain radian, explorating downward and meanwhile touching dilator bougies by left hand. The diagnostic foundation of the stenosis of type II is, on the basis of obvious dilatation of proximal chledochus (diameter $\geq 15 \mathrm{~mm}$ ), to eliminate the other factors, such as stone, tumor as well, and to prove that pancreas exist really inflammation. The followings must be provided: 1) The bougie should clearly be touched at the the back of the pancreas, or by turning over the duodenum, the compressed state of the bile duct in its pancreatic part could be seen; 2) Bake's dilators bigger than No.6 and No.14 French urinary acatheter can be used to pass through the choledochal terminal. By comparison, above-mentioned conditions is unrequired to the stenosis of type I.

Type III is pseudocystic oppression stenosis (8.5\%). Pancreatic pseudocysts in the head can construct directly the bile duct to stricture [25]. According to whether complicating or not the stenosis of type I or II, the type can be divided into three subtypes. Type IIIo is the stenosis merely due to cystic compression, which is seldorn seen in clinical, only 1 case in the group. Type IIIa is the stenosis due to cystic compression with type I, and there are two cases in the group. Type IIIb is the stenosis due to cystic compression with type II, and only 1 case in the group.

As a matter of fact, type III is a mixed type stenosis caused mainly by pseudocyst, and could be found by 
means of B-ultrasoniography and CT. There is more difficult in preoperative diagnosis of the stenosis of type I or II. In the light of the principle of hypromechanics, we know that more serious stenosis in the end-piece biliary duct is, higher press in the proximal biliary duct is, and more distinct dilatation in the proximal biliary duct is. In that stenotic degree of type $\mathrm{I}$ is more serious than one of type II, its dilatation in the proximal bile duct is more obvious, and there is a notable discrepancy in two types ( $\mathrm{P}=0.0018)$. As is known in the Table 2, it could be seen that $76.9 \%$ of paticents belongs to the stenosis of type I if proximal choledochus is dilated, and its diameter is more than $20 \mathrm{~mm}$. And it could identify its stenosis of type I that its choledochal diameter is more than $23 \mathrm{~mm}$. $57.4 \%$ of patients belongs to the stenosis of type II if proximal choledochal diameter is below $20 \mathrm{~mm}$. Certainly, it is necessary to take a dynamic observationof pathologic change. AKP examination is an indictive index to diagnose the obstruction of biliary tract. And AKP values of all patients in the group are raised with different degree. From the Table 3, it could be seen that there is notable different AKP value between the stenosis of type I and II $(\mathrm{P}=0.0121)$. If AKP value is over four times than the upper limit of normal reference value, it could be diagnosed the stenosis of type I.

\subsection{Treatment}

Most of patients with choledochal end-piece stenosis due to biliogenic CP need surgical management, that is cholecystectomy, besides exploratory operation to clear away calculi in bile duct, and controlling development of their diseases as far as possible. In narcosis, we regard habitually it as safe index that the Bake's dilators more than No. 4 or/and the French urinary catheters more than No. 12 can get through the choledochal end-piece. If only the Bake's dilators smaller than No. 3 and the French urinany catheters smaller than No. 10 can get through, it shows there is serious stenosis in the choledochal endpiece [26] that need treatment to avoid occurring acute cholangitis and biliaty cirrhosis of liver.

Type I should be treated with internal drainage owing to serious stenosis, simple cholecystojejunostomy or choledochodedenostomy and with cholangitis, and oddi's spnincterotomy and spnincteroplasty is easy to defeat on account of stenostic segmentofover $30 \mathrm{~mm}$ [27]. Frey et al. [8] had reported that $66 \%-100 \%$ if patients with sphincterotomy to treat choledochal terminal stenosis due to $\mathrm{cp}$ often required to be reoperated so it should be carefal to choose operation program. Ideal choice is Roux-en-x-type choledochojejunestomy [28]. If the stenosis is caused byalcholicor idiopathic CP, without pancreatic calculi and pseoducysts, it can be replaced internal by endoscopy treatment [29-36]. Both surgery and endoscopy are effective methods to cure choledochal terminal stenosis [37-39].

13 patients of type II have only undergone T-tube drainage owing to slighter stenosis and T-tube drainage owing to slighter stenosis and clinical symstom. All had not untoward effect but one had a secondary operation (internal drainage) after pancreatic surgery whose pancreatitis seizure frequently, with jaundice in the stage of each attack. The case might associate with the following factors: 1) pathophysiolagic changes had taken place. $85.1 \%$ of these patients also had gallalader stones at the same time all of them had chelecy stitis. $80 \%$ of patients' bacteriod culture of bile extracted inoperation proved to be positive. These had direct bearing on pathopoiesis and recurrence of their pancreatitis. Atrer the cholecystectomy was conducted, the pollution. Source from focal part was elirninated and the bile was purified, which was favorable to a recovery of pancreatitis. The compression of the pancreas to its common bile duct was consequently lightened. 2) By explorating the cornmon bile duct, the adhesive tissues on the back of the pancreas became loose and separated, therefore, it could lighten, even eliminate the pull to the posterior wall of the common bile duct. 3) Bake's metal dilator itself had a function of the mechanical dilatation. These patients after, operations were routinly given large dose antimicrobials over a week, and the inflam mation in their pancreta had been cured. So it is very safe and effective to use T-tube drainage as a treatment for the stenosis of type II. Since recurrence of pancreatitis could increase the degree of stenosis, T-tube drainage is inapplicable to the patients whose CP have a tendentiousness towards recurrence. But this kind of patient, after all, is minority, only one in the group. Stahl [10] reported that in the course of follow-up to 20 patients, who had had non-operative treatment, only one had undergone biliary tract internal drainage after 3.8 years. Thus it can be inferred that a patient with the stenosis of type II can be treated with non-operative ways, without other illness complicated such as illness of biliary tract, chronic recurrent pancreatitis. Pancreatic duct dilatation and pancreatic pseudocysts, but he must be subjected regularly to the following examinations B-ultraonography, computer tomography and biochemical examinations concerned, besides, the change of his condition should be observed closely.

The patients with the stenosis of type III should undergo cyst internal drainage. Warshaw [40] considered that relying merely on internal drainage to reduce pressure due to cyst, the obstruction of choledochus due to fibrosis formation couldn't often be relieved, so in operation, the adhesive tissues on the back of the pancreas should be freed and relaxed to reduce the pull to the biliary duct. After lysis, the patients with the stenosis of type IIIo or IIIb can undergo cyst internal drainage at the 
same time T-tube placement in choledochus. To the patients with type IIIa, the internal drainage should be undergone in the pseudocyst as well as expansive choledochus, and double Roux-en-Y anastomosis could be adopted [41]. Cut a section of jejunum with mesentery blood vessel, about $15 \mathrm{~cm}$ length. Its proximal end is occluded, its lateral wall is anastomosed with cyst, and its distal end is stitched with former distal jejunum's end, which is connected with biliary duct. Then in the lower part of it (about $50 \mathrm{~cm}$ from choledocho-jejunal anastomotic sto$\mathrm{ma}$ ), it is anastomosed with the proximal end of former jejunum. The procedure takes time and energy, but it can reduce the reflux of in restinal juice.

The patient, whose pancreatic duct has an expanded segment more than $7-8 \mathrm{~mm}$, should be subjected to Puestow's operation program [42]. The patients with duodenal obstruction, lasting more than 3 - 4 months, should undergo gastrojejunostomy. Kamal, Prochorov et al. suggest that the best choice to a patient, who suffers from choledochal duodena, and pancreatic lesions, is pancreatoduodenectomy $[43,44]$.

\section{Significance}

Significance of this research lies in that:

1) This article has originally put forward the theory that there are three types of choledochal end-piece stenosis due to CP. These not only have explained some clinical pictures of $\mathrm{CP}$, but also have instructive significance to diagnose and treat the choledochal end-piece stenosis.

2) From the aspects of anatorny and pathology, the authors have demonstrated cause and diagnostic methods to three types of stenosis.

3) The authors have named three type of stenosis, that is, the external-pressing annular stricture, the front wall of choledochus being compressed stenosis and pseudocystic oppression one. Moreover, according to the anatomic characters of type III, it has been divided into three subtypes (IIIo, IIIa, IIIb).

4) As far as the treatment is concerned, the authors have come up with different methods to treat different stenoses. Besides, the authors have introduced clearly the difference between the stenosis due to biliogenic CP and ones due to non-biliogenic $\mathrm{CP}$, namely, most of the patients with the former need undergo surgical treatment.

\section{Remaining Questions}

This research has put forward the theory that there are three types of choledochal end-piece stenosis due to $\mathrm{CP}$ as well as diagnostic methods, mainly according to explorations of operation and anatomy of corpses. However, before operation, how to diagnose and make differential diagnose better precisely is still a question worthwhile to be studied.

\section{REFERENCES}

[1] C. E. Morrow, J. I. Cohen, D. E. Sutherland and J. S. Najarian, "Chronic Pancreatitis: Long-Term Surgery Results of Pancreatic Duct Drainage, Pancreatic Resection, and near Total Pancreatomy and Islet Autotransplantation," Surgery, Vol. 96, 1984, p. 608.

[2] J. S. Aldrete, H. Jimenez and N. B. Halpern, "Evaluation and Treatment of Acute and Chronic Pancreatitis," Annals of Surgery, Vol. 191, 1980, p. 664.

[3] C. C. Lin, H. P. Wang, M. F. Chen, M. S. Soon, L. R. Mo, X. Z. Lin, S. H. Lin, T. H. Yang, T. H. Wang and J. T. Lin, "Chronic Calcifying Pancreatitis in Taiwan: A Multicentric Study and Comparison with Western Countries," Hepato Gastroenterology, Vol. 44, No. 15, 1997, pp. 842848.

[4] K. Shen, "Characteristics and Surgical Treatment of Chronic Pancreatitis in China," Chinese Journal of Practical Surgery, Vol. 17, No. 4, 1997, pp. 193-194.

[5] T. J. Stahl, M. O. Allen, H. J. Ansel and J. A .Vennes, "Partial Biliary Obstruction Caused by Chronic Pancreatitis," Annals of Surgery, Vol. 207, No. 1, 1988, pp. 26-32. doi:10.1097/00000658-198801000-00007

[6] W. J. Schulte, A. J. LaPorta, R. E. Condon, G. F. Unger, J. E. Geenen and J. J. De Cosse, "Chronic Pancreatitis: A Cause of Biliary Stricture,” Surgery, Vol. 82, 1977, pp. 303-309.

[7] R. T. Ran, "Surgical Anatomy of Choledochal End-Piece," Common Surgery of Clinic (CN), Vol. 3, 1986, pp. 200203.

[8] F. Wishooff, J. Jakobsen and M. Osnes, "Stenosis of the Common Bile Duct in Chronic Pancreatitis," British Journal of Surgery, Vol. 69, No. 1, 1982, pp. 52-54. doi:10.1002/bjs. 1800690117

[9] J. E. da Cunha, T. Bacchella, C. B. Mott, J. Jukemura, E. E. Abdo and M. C. Machado, "Surgical Treatment of Biliary Complications from Calcifying Chonic Pancreatitis," International Surgery (US), Vol. 69, No. 2, 1984, pp. 149-154.

[10] B. E. Stabile, R. Calabria, S. E. Wilson and E. Passaro Jr., "Structure of the Common Bile Duct from Chronic Pancreatitis," Surgery Gynecology \& Obstetrics, Vol. 165, No. 2, 1987, pp. 121-126.

[11] L. Pereira-Lima, A. N. Kalil and T. J. Wilson, "Surgical Treatment of Chronic Pancreatic Cholangiopathy," British Journal of Surgery, Vol. 76, No. 11, 1989, pp. 11291131. doi:10.1002/bjs. 1800761107

[12] J. Devière, S. Devaere, M. Baize and M. Cremer, "Endoscopic Biliary Drainage in Chronic Pancreatitis," Gastrointestinal Endoscopy, Vol. 36, No. 2, 1990, pp. 96100. doi:10.1016/S0016-5107(90)70959-5

[13] C. F. Frey, M. Suzuki and S. Isagi, "Treatment of Chronic Pancreatitis Complicated by Obstruction of the Common Bile Duct or Duodenum," World Journal of Surgery, Vol. 14, 1990, pp. 59-69. doi:10.1007/BF01670547

[14] R. M. Kaikaus and J. E. Geenen, "Current Role of ERCP in the Management of Benign Pancreatic Disease," Endoscopy, Vol. 1, 1997, pp. 111-114.

[15] K. M. Itani and T. V. Taylor, "The Challenge of Therapy 
for Pancreatitis Related Common Bile Duct Stricture," The American Journal of Surgery, Vol. 170, No. 6, 1995, pp. 543-546. doi:10.1016/S0002-9610(99)80012-9

[16] R. A. Prinz, G. V. Aranha, H. B. Greenlee and D. M. Kruss, "Common Duct Obstruction in Patients with Intractable Pain of Chronic Pancreatitis," American Journal of Surgery, Vol. 48, No. 8, 1982, pp. 373-377.

[17] H. J. Sugerman, G. R. Barnhart and H. H. Newsome, "Selective Drainage for Pancreatic,biliary, and Duodenal Obstruction Secondary to Chronic Fibrosing Pancreatitis," Annals of Surgery, Vol. 203, No. 5, 1986, pp. 558-567. doi:10.1097/00000658-198605000-00017

[18] A. L. Warshaw, "Conservation of Pancreatic Tissue by Combined Gastric, Biliary and Pancreatic Duct Drainage for Pain from Chronic Pancreatitis," American Journal of Surgery, Vol. 149, No. 4, 1985, pp. 563-569. doi:10.1016/S0002-9610(85)80057-X

[19] I. Segal, H. H. Lawson, B. Rabinowitz and D. G. Hamilton, "Chronic Pancreatitis and the Hepatobiliary System," American Journal of Surgery, Vol. 77, No. 11, 1982, pp. 867-874.

[20] W. K. Huizinga, S. R. Thomson, J. M. Spitaels and A. E. Simjee, "Chronic Pancreatitis with Biliary Obstruction," Annals of the Royal College of Surgeons of England, Vol. 74, No. 2, 1992, pp. 119-123.

[21] J. H. Tan, F. V. Coakley, Z. J. Wang, L. Poder, E. Webb and B. M. Yeh, "Pseudotumor of the Distal Common Bile Duct at Endoscopic Retrograde Cholangiopancreatography," Clinical Imaging, Vol. 35, No. 4, 2011, pp. 279283.

[22] S. Nagazima, K. Yasuda and S. Huzimoto, "Tankan Mattan bu Ni Okeru Kyosakusyo," Izyotyo, Vol. 16, No. 11, 1981, pp. 1185-1189.

[23] B. B. Newton, M. S. Rittenbury and M. C. Anderson, "Extrahepatic Biliary Obstruction Associated with Pancreatitis," Annals of Surgery, Vol. 197, No. 6, 1983, pp. 645-653. doi:10.1097/00000658-198306000-00002

[24] Y. F. Lu, X. X. Zhang and Y. H. Dong, "Chronic Pancreatitis-Induced Compressed Relative Stenosis of the Distal Common Bile Duct," Hepatobiliary \& Pancreatic Diseases International, Vol. 5, No. 1, 2006, pp. 119-122.

[25] A. Kuroda, T. Konishi, W. Kimura, H. Nagai, M. Sugiyama, D. Semba, Y. Atomi, Y. Morioka and T. Muto, "Cystopancreaticostomy and Longitudinal Pancreaticojejunostomy as a Simpler Technique of Combined Drainage Operation for Chronic Pancreatitis with Pancreatic Pscudocyts Causing Persistent Cholestasis," Surgery Gynecology \& Obstetrics, Vol. 177, No. 2, 1993, pp. 183-186.

[26] J. S .Li, M. C. Wu and Z. Q. Huang, "Surgerey, Common Surgery," Publishing House of People's Army Medicine. 1996, pp. 701-702.

[27] F. E. Eckhauser, J. A. Knol, W. E. Strodel, S. Achem and T. Nostrant, "Common Bile Duct Strictures Associated with Chronic Pancreatitis," American Journal of Surgery, Vol. 49, No. 7, 1983, pp. 350-358.

[28] A. M. Cooperman, "Surgical Treatment of Pancreatic Pseudocyst," Surgical Clinics of North America, Vol. 81, No. 2, 2001, pp. 411-419.

\section{doi:10.1016/S0039-6109(05)70129-8}

[29] J. Deviere, M. Baize, A. Vandermeeren, M. Buset, M. Delhaye and M. Cremer, "Endoscopic Stenting for Biliary Strictures," Acta Gastro-Enterologica Belgica, Vol. 55, No. 3, 1992, pp. 295-305.

[30] M. Barthet, J. P. Bernard, J. L. Duval, C. Affriat and J. Sahel, "Biliary Stenting in Benign Biliary Stenosis Complicating Chronic Calcifying," Endoscopy, Vol. 26, No. 7, 1994, pp. 569-572. doi:10.1055/s-2007-1009041

[31] J. Deviere, M. Cremer, M. Baize, J. Love, B. Sugai and A. Vandermeeren, "Management of Common Bile Duct Stricture Caused by Chronic Pancreatitis with Metal Mesh Self Expandable Stents," Gut, Vol. 35, No. 1, 1994, pp. 122-126. doi:10.1136/gut.35.1.122

[32] C. B. Ross, W. Z. H'Doubler, K. W. Sharp and J. R. Potts, "Recent Experience with Benign Biliary Strictures," American Journal of Surgery, Vol. 55, No. 1, 1989, pp. 64-70.

[33] D. L. Cahen, E. A. Rauws, D. J. Gouma, P. Fockens and M. J. Bruno, "Removable Fully Covered Self-Expandable Metal Stents in the Treatment of Common Bile Duct Strictures Due to Chronic Pancreatitis: A Case Series," Endoscopy, Vol. 40, No. 8, 2008, pp. 697-700. doi:10.1055/s-2008-1077353

[34] Y. Sakai, T. Tsuyuguchi, T. Ishihara, S. Yukisawa, H. Sugiyama, K. Miyakawa, Y. Kuroda, T. Yamaguchi, S. Ozawa and O. Yokosuka, "Long-Term Prognosis of Patients with Endoscopically Treated Postoperative Bile Duct Stricture and Bile Duct Stricture Due to Chronic Pancreatitis," Journal of Gastroenterology and Hepatology, Vol. 24, No. 7, 2009, pp. 1191-1197. doi:10.1111/j.1440-1746.2009.05878.x

[35] D. M. Tan and S. Sherman, "Endoscopic Therapy in Chronic Pancreatitis," Korean Journal of Internal Medicine, Vol. 26, No. 4, 2011, pp. 384-399. doi:10.3904/kjim.2011.26.4.384

[36] A. Katanuma, H. Maguchi, M. Osanai and K. Takahashi, "Endoscopic Ultrasound-Guided Biliary Drainage Performed for Refractory Bile Duct Stenosis Due to Chronic Pancreatitis: A Case Report," Digestive Endoscopy, Vol. 24, Suppl. 1, 2012, pp. 34-37. doi:10.1111/j.1443-1661.2012.01256.x

[37] P. Cantù, L. C. Hookey, A. Morales, O. Le Moine and J. Devière, "The Treatment of Patients with Symptomatic Common Bile Duct Stenosis Secondary to Chronic Pancreatitis Using Partially Covered Metal Stents: A Pilot Study," Endoscopy, Vol. 37, No. 8, 2005, pp. 735-739. doi:10.1055/s-2005-870130

[38] M. E. Smits, E. A. Rauws, T. M. van Gulik, D. J. Gouma, G. N. Tytgat and K. Huibregtse, "Long-Term Results of Endoscopic Stenting and Surgical Drainage for Biliary Stricture Due to Chronic Pancreatitis," The British Journal of Surgery, Vol. 83, No. 6, 1996, pp. 764-768.

[39] M. Delhaye, M. Arvanitakis, M. Bali, C. Matos and J. Devière, "Endoscopic Therapy for Chronic Pancreatitis," Scandinavian Journal of Surgery, Vol. 94, No. 2, 2005, pp. 143-153.

[40] A. L. Warshaw, R. H. Schapiro, J. T. Ferrucci Jr and J. J. Galdabini, "Persistent Obstructive Jaundice, Cholangitis, and Biliary Cirrhosis Due to Common Bile Duct Stensis 
in Chronic Pancreatitis," Gastroentorology, Vol. 70, No. 4, 1976, pp. 562-567.

[41] J. M. Regimbeau, D. Fuks, E. Bartoli, M. Fumery, A. Hanes, T. Yzet and R. A. Delcenserie, "Comparative Study of Surgery and Endoscopy for the Treatment of Bile Duct Stricture in Patients with Chronic Pancreatitis," Surgical Endoscopy, Vol. 26, No. 10, 2012, pp. 29022908. doi:10.1007/s00464-012-2283-7

[42] T. Aimoto, E. Uchida, Y. Nakamura, K. Yamahatsu, A. Matsushita, A. Katsuno, K. Cho and M. Kawamoto, "Current Surgical Treatment for Chronic Pancreatitis," Journal of Nippon Medical School, Vol. 78, No. 6, 2011,

\section{pp. 352-359. doi:10.1272/jnms.78.352}

[43] H. J. Sugerman, G. R. Barnhart and H. H. Newsome, "Selective Drainage for Pancreatic, Biliary, and Duodenal Obstruction Secondary to Chronic Fibrosing Pancreatitis," Annals of Surgery, Vol. 203, No. 6, 1986, pp. 558567. doi:10.1097/00000658-198605000-00017

[44] A. V. Prochorov, K. J. Oldhafer, S. I. Tretyak, S. M. Rashchynski, M. Donati, N. T. Rashchynskaya and D. A. Audzevich, "Surgical Treatment of Pain in Patients with Chronic Pancreatitis," Hepato-gastroenterology, Vol. 59, No. 116, 2012, pp. 1265-1269. 